\title{
Plasma Assisted Oblique Angle Deposition of Transparent and Conductive in- Plane Anisotropic ITO Thin Films
}

\author{
${ }^{\mathrm{a}}$ J. Parra-Barranco, ${ }^{\mathrm{a}} \mathrm{J}$. R. Sanchez-Valencia, ${ }^{\mathrm{a}} \mathrm{F}$. J. Aparicio, ${ }^{\mathrm{a}} \mathrm{F}$. Garcia-Garcia, ${ }^{\mathrm{b}}$ F. J. \\ Ferrer, ${ }^{\mathrm{a}}$ V. Rico, ${ }^{\mathrm{a}} \mathrm{C}$. Lopez-Santos, ${ }^{\mathrm{a}} \mathrm{A}$. Borras, ${ }^{\mathrm{a}} \mathrm{A}$. R. Gonzalez-Elipe and ${ }^{\mathrm{a}} \mathrm{A}$. Barranco \\ ${ }^{a}$ National Research Council. Materials Science Institute of Sevilla (CSIC-US) c/Américo \\ Vespucio 49, 41092 Sevilla. Spain \\ ${ }^{\mathrm{b}}$ Centro Nacional de Aceleradores (CSIC-Universidad de Sevilla) Av. Thomas A. Edison, \\ 7, 41092 Sevilla, Spain
}

\begin{abstract}
Oblique angle deposition (OAD) is a powerful technique for the fabrication of porous nanostructured oxide thin films. OAD films typically present a columnar tilted nanostructure due to geometrical shadowing effects during the thin film growth. In this work, we study the fabrication of transparent and conducting indium tin oxide films (ITO) by OAD assisted by a microwave ECR plasma. The objective of assisting the deposition with a plasma discharge is to modify the growth mechanism of the OAD process introducing additional parameters to control the columnar microstructure, composition, porosity of the films. The results indicate the OAD ITO deposition assisted by the plasma discharge is a very effective process to develop in-plane structural anisotropy in the ITO nanocolumnar films what determines their electrical properties.
\end{abstract}

\section{Introduction}

Indium tin oxide (ITO) is the most studied and utilized transparent conducting oxide (TCO) in a very wide range of technological applications (1). ITO thin films have been prepared using different deposition techniques including wet (i.e., sol-gel, dip-coating, etc.) and dry,(i.e., thermal and e-beam evaporation, magnetron sputtering, etc.). Recently, porous nanostructured ITO thin films and multilayers have been prepared by deposition at oblique angles (OAD) and Glancing Angle Deposition (GLAD) (2). In these manufacturing processes, the substrates form an oblique angle with respect to the flux direction of the evaporated or sputtered precursor material. At present, many different nanostructured porous thin films of oxides, semiconductors, polymers, metals and molecular materials have been obtained by oblique angle deposition techniques. Their microstructures basically consist of tilted nanocolumns what confer to the films their outstanding properties $(2,3)$.

OAD-ITO films have been stacked in the form of 1D photonic crystals, and deposited forming branched ITO nanostructures under conditions inducing a vacuum liquid-solid growth $(4,5)$. In these layers, the electrical conductivity might be different along different directions basically due to a reduced lateral connectivity between the nanostructures.

In this work, we present an experimental strategy consisting of the use of remote plasmas to assist the growth of e-beam evaporated ITO thin films. We describe here the experimental procedure and present a detailed characterization of the ITO films prepared both by direct e-beam evaporation (OAD ITO films) or by assisting the deposition with 
Ar or $\mathrm{O}_{2}$ remote plasma discharges (Plasma OAD ITO films). Field emission scanning electron microscopy (SEM), topographic and conducting atomic force microscopy (AFM), and conductivity measurements have been utilized for the characterization of the samples. We study the effects of the plasma assisted deposition on the nanostructure of the films and their optical and electrical properties.

\section{Experimental Procedure}

\section{Thin Film Preparation}

ITO thin films have been deposited in an e-beam evaporator system previously described for the OAD of nanostructured porous oxide thin films. (6-8) In this system, a large number of substrates can be deposited simultaneously at different zenithal angles $(\alpha)$ of evaporation (Figure 1). Values of $\alpha=60^{\circ}, 70^{\circ}, 80^{\circ}$ and $85^{\circ}$ have been used for the films deposited in the absence of plasma (Figure 1), while only $\alpha=80^{\circ}$ was used when assisting their growth with a plasma. This value of $\alpha$ is determined by the position of the plasma source. The experimental set-up is shown in Figure 1.

The plasma source was a electron cyclotron resonance (ECR) microwave source (2.45 $\mathrm{GHz}$ ) operating at $460 \mathrm{~W} \cdot \mathrm{O}_{2}$, Ar were used as plasma gas. OAD and Plasma OAD films were grown at the same deposition pressure of over $4 \times 10^{-4}$ torr. The evaporation rates were over 0.7-1.0 $\AA \mathrm{s}^{-1}$ determined with a quartz crystal monitor at the sample holder position.

ITO Pellets supplied by Kurt J. Lesker $\left(\mathrm{In}_{2} \mathrm{O}_{3} / \mathrm{SnO}_{2} 90 \% / 10 \%(\mathrm{w} / \mathrm{w})\right)$ were placed in a graphite crucible for the e-beam evaporation. The thin films were deposited on $\mathrm{Si}(100)$ wafers and quartz and ITO plates for the different characterization essays.

\section{Thin Film Characterization}

The microstructure of the films was studied in samples deposited on Si (100) wafer pieces using a field-emission scanning electron (FESEM) microscope HITACHI-S-5200 operated at $2 \mathrm{KV}$. Coated wafer pieces were cleaved for the cross-sectional examination. The optical properties of the films deposited on either quartz or ITO plates were determined by UV-vis transmission spectroscopy with a PerkinElmer Lambda 750 spectrophotometer in the range 190-3500 nm.

The composition of the coatings was evaluated at the $3 \mathrm{MV}$ NEC 9SDH-2 tandem accelerator of the National Center for Accelerators (CNA, Seville, Spain) Rutherford Backscattering Spectroscopy (RBS), using a beam of $2.0 \mathrm{MeV}$ and a passivated implanted planar silicon (PIPS) detector set at $165^{\circ}$. Sn/In ratios were obtained by Particle Induced X-ray Emission (PIXE) using a $3.0 \mathrm{MeV}$ proton beam and a $\mathrm{Si}(\mathrm{Li}$ ) detector set at $135^{\circ}$. Atomic force microscopy (AFM) characterization of the film surface topography was carried out with a Nanotec Dulcinea AFM microscope. Scanning conductivity measurements were obtained with a current sensing module supplied with a conductive tip NT-MDT with a gold cover. The diameter of the conductive tip was $70 \mathrm{~nm}$ and it presented a resonance frequency of $190-325 \mathrm{KHz}$ and a force constant of 5.5-22.5 $\mathrm{N} / \mathrm{m}$.

\section{Electrical Measurements}


Cross section and sheet resistance measurements were carry out with a Keithley 2635A SourceMeter. The OAD thin films were deposited on commercial ITO plates for the cross sectional measurements. In this case, the conductive ITO substrates and copper contacts on the surface were the electrodes for the electrical measurements. Films grown on a quartz plate were used for sheet measurements. In this case, two copper contacts were placed in the sample surface consisting in circular conductive adhesive spots of $3 \mathrm{~mm}$ diameter separated by $8 \mathrm{~mm}$. The intensity-voltage (I-V) curves were obtained with a compliance of $10^{-3} \mathrm{~A}$. and a voltage range of $\pm 4 \times 10^{-3} \mathrm{~V}$.
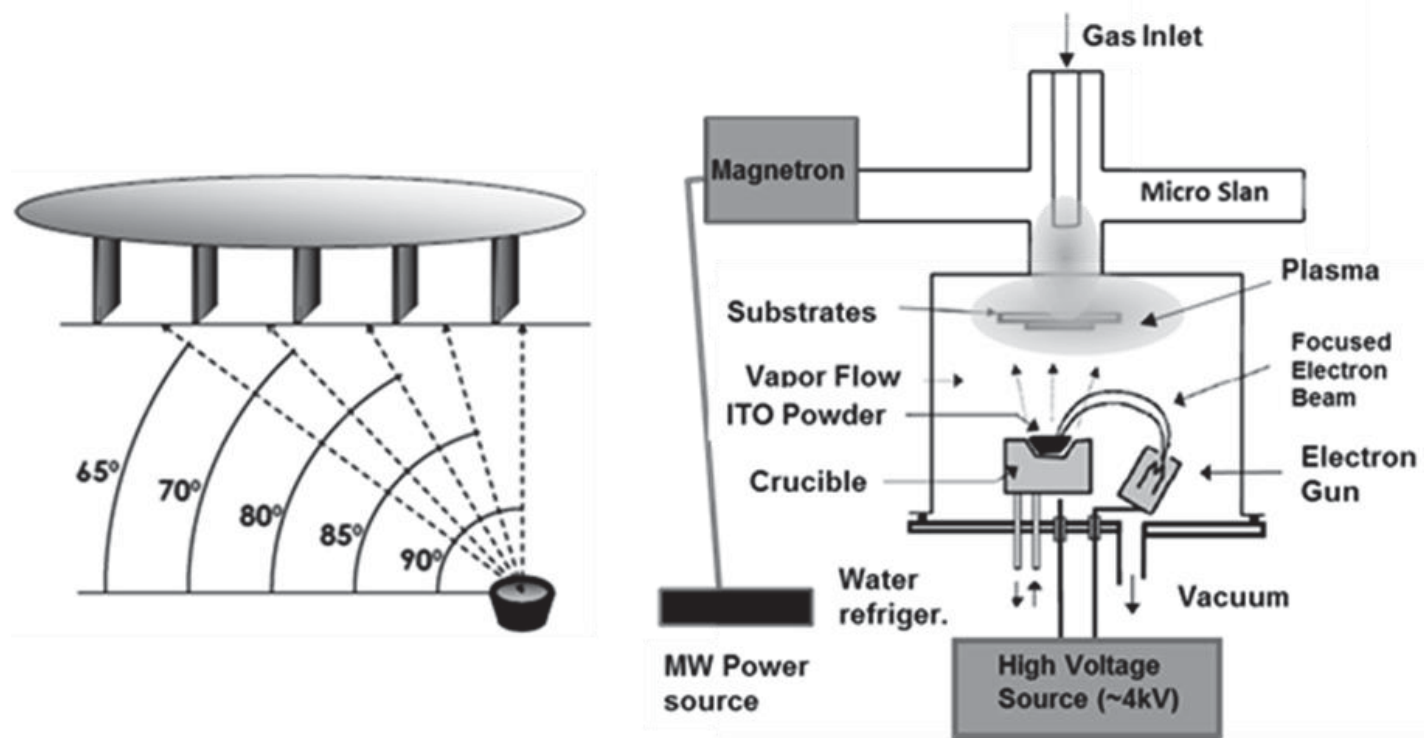

Figure 1. Geometry of the OAD deposition and scheme of the experimental set up used for the e-beam deposition of ITO films in OAD configuration while assisting their growth with a downstream plasma

\section{Results and Discussion}

Figure 2a shows normal and cross section SEM micrographs of a series of ITO OAD thin films prepared at $\alpha=80^{\circ}$ under a pressure of $10^{-4}$ torr of $\mathrm{O}_{2}$. The micrographs show that the evaporated material aggregates in the form of individual tilted nanocolumns extending from the interface with the substrate to the surface of the film. These nanocolumns slightly bend to higher angles with respect to the perpendicular to the surface as the film thickness increases. A rough estimation of the average nanocolumnar tilting angle $(\beta)$ was over $45^{\circ}$. This value of the tilting angle do not agree well with the predictions of the commonly accepted tangent rule for OAD deposition processes. We have recently explained this discrepancy assuming a trapping process for the ballistic particles flying close to the deposited nanostructures (9). Another notable feature of these OAD ITO nanostructures is that they are rather smooth and do not show the typical feather-like termination profile characteristic of $\mathrm{TiO}_{2}$ and other oxides evaporated in an oblique angle configurations $(2,6,7)$. Other authors have obtained similar thin film microstructures for e-beam OAD ITO films (10). 
a)
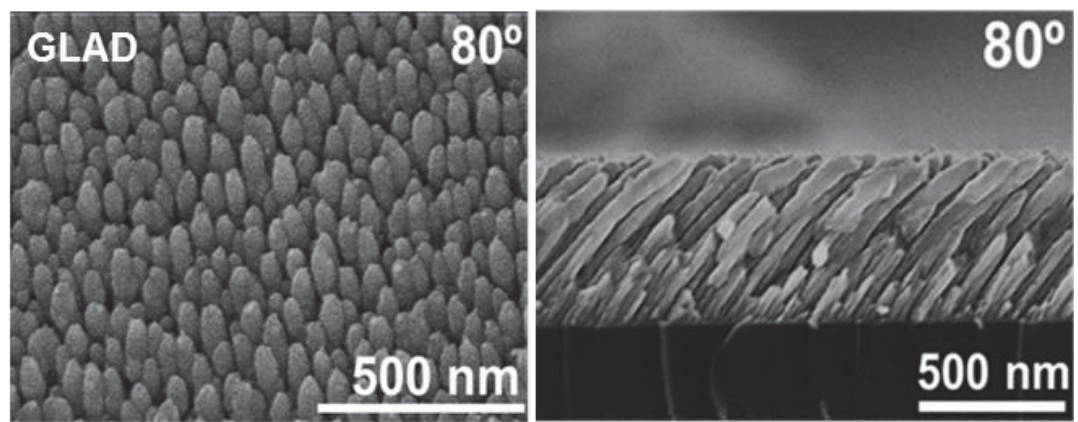

b)
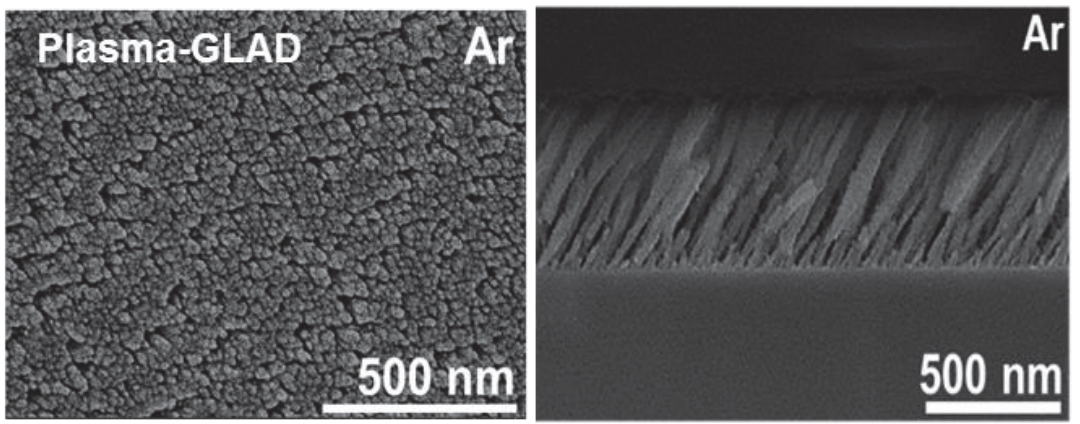

c)
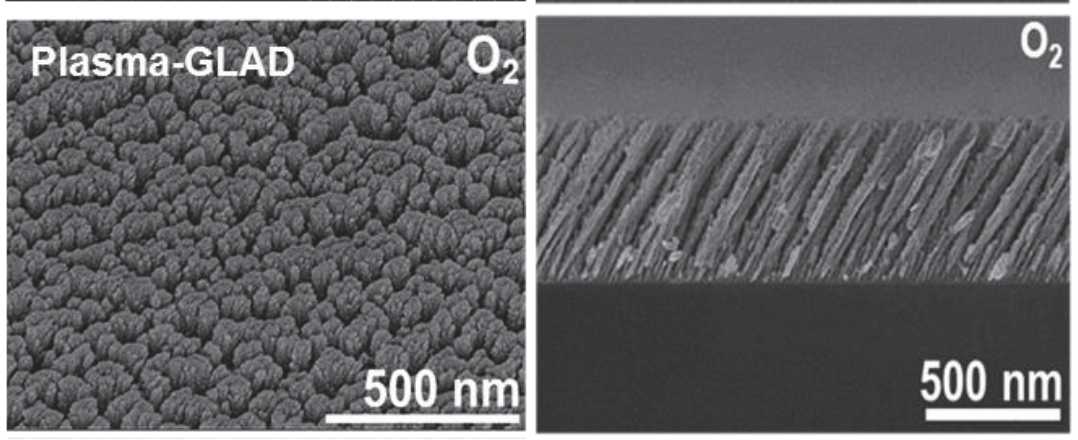

Figure 2. Planar view and cross-sectional FESEM micrographs of ITO thin films deposited by (a) OAD at $80^{\circ}$ (b) Oxygen plasma-OAD at $80^{\circ}$ (c) Oxygen plasma-OAD at $80^{\circ}$.

Figure $2 \mathrm{~b}$ shows a series of normal and cross-sectional SEM micrographs corresponding to ITO thin films prepared at $\alpha=80^{\circ}$ in the presence of $\mathrm{Ar}$ and $\mathrm{O}_{2}$ plasmas. Two conclusion can be extracted from the FESEM micrographs. The first is the ECR plasma conditions are compatible with the OAD process. Thus, the ITO films deposited by plasma-OAD present the typical tilted columnar microstructure characteristic of the oblique angle deposition processes. The second conclusion is the morphology of the films (Figure 2b and 2c) experienced significant changes affecting to the shape, size, agglomeration and tilting angle of the nanocolumns. Thus, the nanocolumns of the Plasma OAD films are not isolated but associate forming columns bundles that stretch along a direction perpendicular to the incoming flux (see the planar view images in Figure 2a to 2c). The bundling association of nanocolumns in OAD thin films is a phenomenon previously reported for different materials (2). In fact, the in-plane anisotropies resulting of the nanolumnar bundling associations are very interesting for the optical applications of OAD films such as optical encryption and the development of switchable gratings and highly dichroic films $(8,11,12)$. Another important characteristic 
of the Plasma OAD ITO thin films is that the tilting angle $\beta$ is over $30^{\circ}$ a value that is lower than the one obtained without plasma, varying slightly with the type of plasma gas. This visual assessment is confirmed by the 2D Fourier transform (FFT) plots of these images (not shown) that are characterized by an asymmetric shape for the plasma OAD thin films and by a symmetric rounded shape for the $80^{\circ}$ thin film deposited in the absence of plasma. This difference sustains that in the plasma-assisted films the surface aggregates are associated forming bundles along a preferential direction perpendicular to the incoming material flux. The AFM analysis of the films clearly confirms the existence of a preferential nanocolumnar bundling association direction in the films deposited by Plasma OAD (data not shown).

The e-beam evaporation of complex materials like ITO powders may induce changes in stoichiometry. The "as-prepared" ITO thin films deposited in the absence of plasma were amorphous when examined by X-ray diffraction and presented a dark coloration indicating a certain lack of oxygen with respect to the stoichiometric mixed oxide. The $\mathrm{O}_{2}$ plasma-assisted films were also amorphous but less dark. Figure 3a shows the UVVIS-NIR spectra of ITO films after annealing in $\mathrm{O}_{2}$ at $350^{\circ} \mathrm{C}$ for 6 hours. The results indicated the annealed films become transparent and crystalline without experiencing any microstructural change observable by FESEM. The shapes of the OAD and plasma-OAD curves were similar presenting the characteristic ITO visible transparency and NIR absorption at wavelengths higher than $1200 \mathrm{~cm}^{-1}$. The X-ray diagrams of the annealed samples (data now shown) were characterized by well-defined peaks attributed to ITO very similar to those of commercial ITO film references. In the case of films deposited using an $\mathrm{O}_{2}$ plasma some small peaks due to $\mathrm{SnO}_{2}$ were detected. Importantly, this minority segregation of tin oxide did not seem to affect significantly the conductivity and optical properties of the films (see below).

The $\mathrm{O} /(\mathrm{In}+\mathrm{Sn})$ atomic ratio and the elemental depth distribution of the films were determined by RBS. The results of this analysis demonstrated that the relative content of oxygen was higher in the plasma-assisted films increasing slightly after annealing. This result is in agreement with the increase in the transparency induced by this treatment as indicated. The relatively higher oxygen content oxygen determined for the plasma assisted films agrees with the enrichment in Sn deduced from the XRD.

Film density values were obtained from the RBS and FESEM characterizations of OAD, Plasma OAD and ITO reference samples. The densities obtained for the plasma assisted OAD films indicated porosities of the order of $40-50 \%$, in agreement with the values of OAD oxide thin films reported in the bibliography $(2,6,7)$.

The annealed films were transparent in the visible and presented a significant absorption in the near infrared. This is a distinctive characteristic of TCO materials attributed to a high concentration of free electrons (1). To characterize the electrical transport properties of the ITO thin films we studied the annealed films by conducting AFM and measured their cross-sectional and sheet conductivities. The conductivity maps (not shown) indicated that most surface features with a high conductivity roughly coincide with those observed in the topographic AFM images. This proves that in the OAD ITO thin films the in-depth conductivity mainly involves a preferential charge transport through the nanocolumns of the films. Moreover, the measured I-V curves at these sites reveal similar local conductivity values for the OAD, plasma-OAD and ITO reference films. A transport pattern approaching an ohmic behavior was obtained in all cases. The similar conductivities at microscopic level between the compact and OAD ITO films indicates that the electronic characteristics of the examined materials are rather similar. However, this does not imply that their macroscopic conductivities are different. 
Macroscopic cross-sectional and "sheet" conductivity measurements were carried out as described in the experimental section. The obtained I-V curves depicted a typical resistive ohmic behavior shown in Figure 3b. The resistivity values obtained show that the in-depth and sheet conductivities of the commercial ITO films used as reference are higher than those of the OAD ITO films. We attribute this difference to the smaller density and the particular nanocolumnar microstructure of the latter samples.
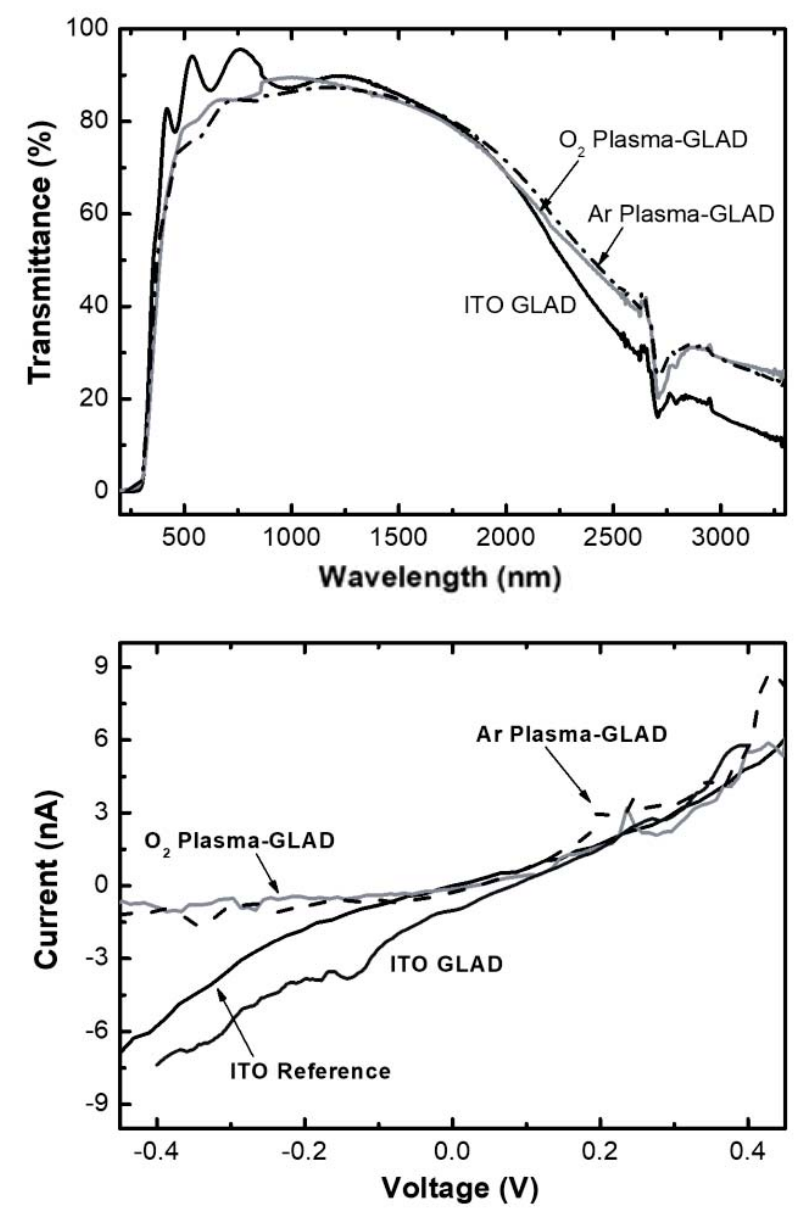

Figure 3. a) UV-Vis transmission spectra of ITO thin films deposited by OAD and Plasma-OAD after the annealing process b) I-V curves of annealed ITO thin films prepared by OAD and Plasma-OAD. The curve of a commercial ITO film reference is included.

The sheet conductivities in the commercial ITO reference films are equivalent along any surface direction. The situation is approximately similar in the case of films deposited by OAD. However, a slight increase in the sheet conductivity of over $14 \%$ was measured along the bundling direction in comparison with the perpendicular direction. In the case of the plasma OAD films, the situation is very different. Thus, differences in conductivities of over $22 \%$ and $80 \%$ were measured along the same axes for the Plasma $\operatorname{Ar} \mathrm{OAD}$ and $\mathrm{O}_{2}$ plasma OAD samples, respectively. These results prove that the bundling direction defined a preferential electrical pathway in the film due to the lateral association of nanocolumns and that this pathway was interrupted in the perpendicular 
direction. The difference was particularly important for the $\mathrm{O}_{2}$ plasma film where the resistivity in the direction perpendicular to the bundling direction was comparatively very high.

\section{Conclusions}

In this work, we demonstrate how the OAD of ITO films is a process compatible with the use of remote plasma discharges. The plasma assistance during the deposition modifies very effectively the nanostructure of the growing films decreasing the nanocolumnar tilting angles and inducing a preferential nanocolumnar bundling association. Both the OAD and the plasma assisted OAD ITO films presented different in-depth and sheet conductivities derived from their particular porous and nanocolumnar structures. The anisotropic in-plane conductivities found in the plasma OAD samples have been associated with preferential conductivity tracks along the bundling direction of the nanocolumns. The possibility of fabricating transparent films showing anisotropic inplane conductivities along well-defined axis opens the way for novel photonic and optoelectronic applications.

\section{Acknowledgments}

We thank the EU (FEDER) and MINECO-AEI for financial support (Projects MAT2016-79866-R, MAT2013-40852-R, MAT2013-42900-P, MINECO-CSIC 201560E055, IJCI-2014-21226, RECUPERA2020. JRS-V and AngelB acknowledge funding from EU project PlasmaPerovSol. This project has received funding from the European Union's Horizon 2020 research and innovation programme under the Marie Skłodowska-Curie grant agreement No 661480.

\section{References}

1. C.G. Granqvist, A. Hultåker, Thin Solid Films, 411, 1 (2002).

2. A. Barranco, A. Borras, A. R. Gonzalez-Elipe, A. Palmero, Progr. Mater. Sci. 76, 59 (2016).

3. M.M. Hawkeye, M.J. Brett, J.Vac. Sci. Technol., A, 25, 1317 (2007).

4. M.F. Schubert, J.K. Kim, S. Chhajed, E.F Schubert, M.J. Ellison, Proc. SPIE, 6674, No. 667403 (2007).

5. R.T. Tucker, A.L. Beaudry, J.M. LaForge, M.T. Taschuk, M.J. Brett, Appl. Phys. Lett., 101, 193101 (2012).

6. L. González-García, A. Barranco, A.M. Páez, A.R. González-Elipe, M.-C. García-Gutiérrez, J.J. Hernández, D.R. Rueda, T.A. Ezquerra, D. Babonneau, ChemPhysChem, 11, 2205 (2010).

7. J.R. Sánchez-Valencia, I. Blaszczyk-Lezak, J.P. Espinós, S. Hamad, A.R. González-Elipe, A. Barranco, Langmuir 25, 9140 (2009).

8. J. Parra-Barranco, F.J. García-García, V. Rico, A. Borrás, C. López-Santos, F. Frutos, A. Barranco, A.R. González-Elipe, ACS Appl. Mater. Interfaces 7, 10993 (2015).

9. R. Alvarez, C. Lopez-Santos, J. Parra-Barranco, V. Rico, A. Barranco, J. Cotrino, A.R. Gonzalez-Elipe, A. Palmero, J. Vac. Sci. Technol., B, 32, 041802 (2014). 
10. D. A., Rider, R. T. Tucker, B. J. Worfolk, K. M. Krause, A. Lalany, M. J. Brett, J. M. Buriak, K. D. Harris, Nanotechnology, 22, 085706 (2011).

11. J. R., Sanchez-Valencia, J. Toudert, A. Borras, A. Barranco, R. Lahoz, G. F. de la Fuente, F. Frutos, A. R. Gonzalez-Elipe, Adv. Mater., 23, 848 (2011).

12. J. Parra-Barranco, M. Oliva-Ramirez, L. Gonzalez-Garcia, M. Alcaire, MaciasMontero, A. Borras, F. Frutos, A. R. Gonzalez-Elipe, A. Barranco, ACS Appl. Mater. Interfaces, 6, 11924 (2014). 